\title{
Numerical Simulation on Acceleration Characteristics of a Supersonic Free Jet through a Plasmajet
}

\author{
Yuka Arai $^{1}$, Yusuke Mizuno ${ }^{1}$, Yu Sumoto ${ }^{2}$, Shun Takahashi ${ }^{3}$, Kota Fukuda ${ }^{1}$ and Hideyuki Horisawa ${ }^{1}$ \\ 1. The Department of Aeronautics and Astronautics, Tokai University, Hiratsuka, Kanagawa 259-1292, Japan \\ 2. Nippon Pneumatic MFG. Co., LTD., Nabari, Mie 518-0605, Japan \\ 3. The Department of Prime Mover Engineering, Tokai University, Hiratsuka, Kanagawa 259-1292, Japan
}

\begin{abstract}
In this study, numerical simulation on acceleration characteristics of a supersonic free jet through utilization of a plasmajet was conducted, where the supersonic free jet was accelerated through an interaction in a shear layer between the free jet and plasmajet. In this analysis, the governing equations including two-dimensional compressible Reynolds-averaged Navier-Stokes equations (RANS) were calculated with a commercial code, ANSYS Fluent v. 14.5. As for the turbulence model, a $k$ - $\varepsilon$ realizable turbulence model was employed. In this paper, when a plasma flow is used for the center nozzle, the velocity is higher especially in potential core part than in the case of unheated flow because of a hot core flow of the plasmajet.
\end{abstract}

Key words: Numerical algorithms, computational fluid dynamics, turbulence modeling, aeroacoustics.

\section{Introduction}

Interactions of several jets have been widely applied in a variety of fields and attracted strong interests for practical applications and theoretical problems because of the complexities of the physical mechanism for interpretation of the flow field, for example, supersonic jet interactions of clustered rocket exhaust plumes for thrust augmentation [1] and of fuel/oxidizer injectors for enhanced mixing through shear layers [2-4]. On the other hand, applications of augmentation of flow velocities have been widely employed for propulsion systems, such as aircraft engines (gas-turbine engines), rocket engines, etc., and for high-speed wind tunnels, as well Refs. [5, 6]. Among them, a regime of electrothermal acceleration, in which a flow is electrically heated, usually by arc discharge, and then aerodynamically accelerated through a nozzle, is primarily used in material processing (as plasmajets) [7, 8], space propulsion

Corresponding author: Yuka Arai, B.Sc. in engineering, research fields: fluid dynamics and aero-thermodynamics. systems (as arcjet thrusters) [6, 9], and arc-heated wind tunnel facilities [10]. With the electrothermal acceleration, since input powers into the flow can be electrically controlled with the control of discharge currents, total temperatures, or namely velocities, can be actively controlled. However, in those devices, the flow of the feedstock is directly heated and then its total temperature is increased. Because of the elevated temperature, use of the flow on surface of materials with low-melting points (or low decomposition temperatures) is not acceptable for material processing, etc.

Therefore, in this study, a novel regime for the acceleration of a supersonic flow with a plasmajet under atmospheric condition is proposed and assessed. Although the above mentioned electrothermal regime employs the direct heating of the flow for acceleration, advantages of the indirect acceleration of the flow with an interaction of the flow with the plasmajet through a shear layer are investigated.

In addition, effects and physical mechanisms of the interaction of the flows through the shear layer for effective acceleration of the flow are to be elucidated. 
In this study, an investigation with a computational fluid dynamic simulation code is conducted on:

(1) influence of number of jets of coaxial, annular, multi nozzles for efficient acceleration of the flow,

(2) potentials on acceleration of a supersonic jet with a plasmajet, and,

(3) optimum operational conditions, of the plasmajet, i.e. total temperatures, exhaust Mach numbers, etc.

\section{Computational Method}

In this study, a commercial CFD solver, ANSYS Fluent v. 14.5, was employed to analyze an axisymmetric Reynolds-averaged Navier-Stokes equations. As for the turbulent model, realizable $k-\varepsilon$ model [11] was employed. The fourth-order Runge-Kutta method and Roe-FDS method were employed for time- and spatial-discretization scheme, respectively.

A simulation model is illustrated in Fig. 1 showing axisymmetric, coaxial, circular, triple nozzle jets. Dimensions of the axisymmetric domain are $150 \mathrm{~mm}$ and $20 \mathrm{~mm}$ in axial ( $x$-direction) and radial (y-direction) directions, respectively. At an inlet boundary (left, at $-x$ ) on the centerline, three inlets of coaxial supersonic jets from a coaxial triple nozzle are placed. The right $($ at $+x$ ) and top $($ at $+y)$ outer boundaries are outlet boundaries, and an axisymmetric boundary for the centerline (at $y=0$ ).

A schematic illustration of the axisymmetric, coaxial, triple supersonic jets is shown in Fig. 2. A diameter of the plasmajet (nozzle 1) labeled as " $D$ " is assumed as a characteristic length of the domain. The width of the smallest grid is $0.05 \mathrm{~mm}$. The main flow subjected to the acceleration with a plasmajet (nozzle 1) is a supersonic jet exhausted from nozzle 2. A jet from the third nozzle (nozzle 3 ) is an assisting jet for the supersonic jet from nozzle 2. A detailed role of nozzle 3 is assessed and described in Section 3.1.

Outlet conditions and a schematic illustration of the coaxial triple supersonic jets are given in Table 1 and

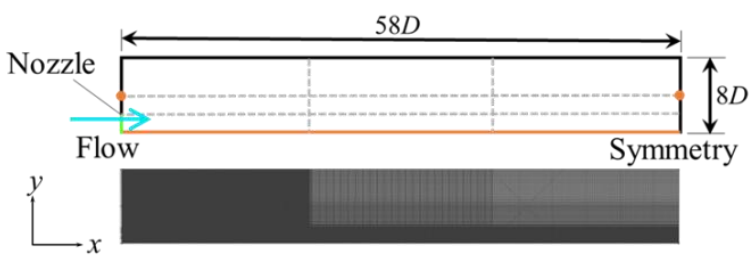

Fig. 1 Simulation model.

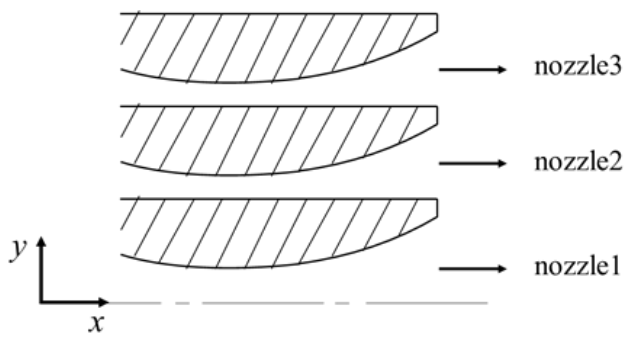

Fig. 2 Schematic illustration of the axisymmetric, coaxial, triple supersonic jets.

Table 1 Outlet conditions of a coaxial triple nozzle for inlet boundaries.

\begin{tabular}{lcccc}
\hline & Nozzle & Mach number & $P_{0} \mathrm{~Pa}$ & $T_{0} \mathrm{~K}$ \\
\hline nozzle 1 & Plasma & 2.65 & & 2000 \\
nozzle 2 & Air & 2 & $0.3 \times 10^{5}$ & 300 \\
nozzle 3 & Air & 1.7 & & 300 \\
\hline
\end{tabular}

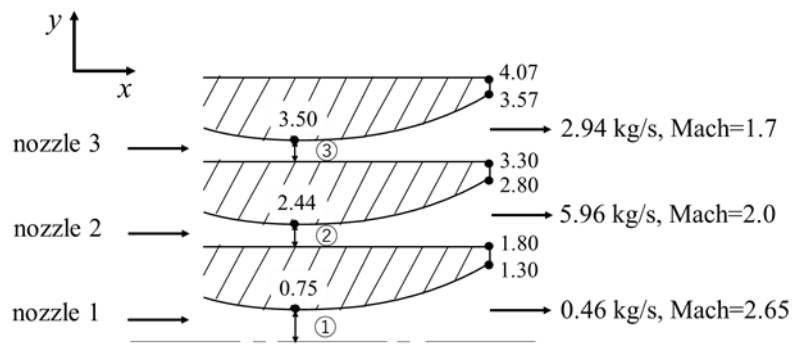

Fig. 3 Schematic illustration of the coaxial triple supersonic jets and conditions.

Fig. 3, respectively. Flows inside and outside of the nozzles are regarded as non-reactive air flows, and a plasmajet is assumed as a high-temperature (or heated) non-reactive air jet, for simplicity.

\section{Computational Results and Discussions}

3.1 The Influence of the Number Nozzles of Coaxial, Annular, Multi Jets

\subsubsection{Conditions}

In this section, an investigation on the influence of number nozzles of coaxial, annular, multi jets was 
Table 2 Flow conditions of the jets from the nozzles.

\begin{tabular}{|c|c|c|c|c|c|c|}
\hline & & \multicolumn{3}{|c|}{ Mach number } & \multirow{2}{*}{$P_{0}, \mathrm{~Pa}$} & \multirow{2}{*}{$T_{0}, \mathrm{~K}$} \\
\hline & & 1 nozzle & 2 nozzles & 3 nozzles & & \\
\hline nozzle 1 & Plasma & 2.65 & 2.65 & 2.65 & & 2000 \\
\hline nozzle 2 & Air & & 2 & 2 & $0.3 \times 10^{5}$ & 300 \\
\hline nozzle 3 & Air & & & 1.7 & & 300 \\
\hline
\end{tabular}

conducted for efficient acceleration of the flow. Flow conditions of the jets from the nozzles, which are labeled as nozzle 1, 2 and 3, are listed in Table 2. The main flow subjected to the acceleration with a plasmajet (nozzle 1) is a supersonic jet exhausted from nozzle 2. A jet from the third nozzle (nozzle 3 ) is an assisting jet for the supersonic jet from nozzle 2 . Comparison of nozzle conditions was conducted among cases of 1 nozzle (plasmajet only), 2 nozzles (plasmajet and annular supersonic jet), and 3 nozzles ( 2 nozzles plus an assisting jet of the third nozzle (nozzle 3)).

\subsubsection{Results and Discussions}

Distributions of velocity magnitude and Mach number at non-dimensional time of $t^{*}=767$ are shown in Figs. $4 \mathrm{a}$ and $4 \mathrm{~b}$, respectively. From the figure, it is shown that larger velocities are further maintained to downstream region with the increase of number of nozzles, or jets.

Distributions of normalized velocity magnitude and kinetic energy, which are normalized by the results of single nozzle case by Eqs. (1) and (2), are shown in Fig. 5 for $x=0.050 \mathrm{~m}$ and $0.100 \mathrm{~m}$ downstream from the nozzles for various nozzle conditions.

$$
\begin{gathered}
\tilde{u}=\frac{u}{u_{\text {case } 1-1}} \\
\tilde{E}=\frac{E}{E_{\text {casel }-1}}
\end{gathered}
$$

From the figures, it is shown that larger velocities and energies can be obtained at central region to jet boundary region $y / d=4$ for $x=0.050 \mathrm{~m}$ and $x=0.100$ $\mathrm{m}$ with the increase of number of jets, or especially with a triple nozzle configuration. Although not shown in these figures, simulation results with various conditions for an assisting jet of nozzle 3 showed that
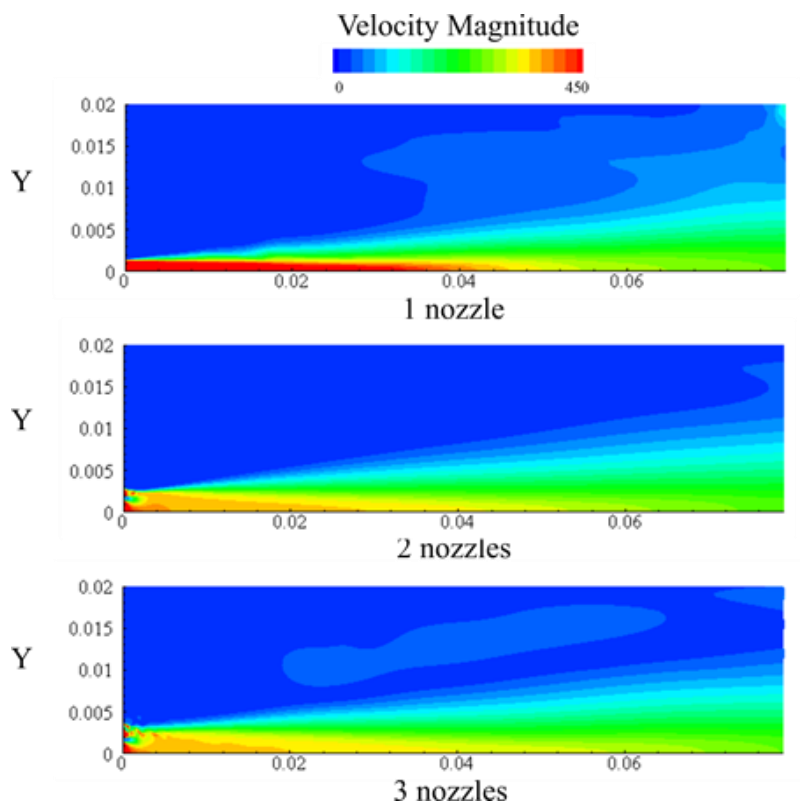

(a)
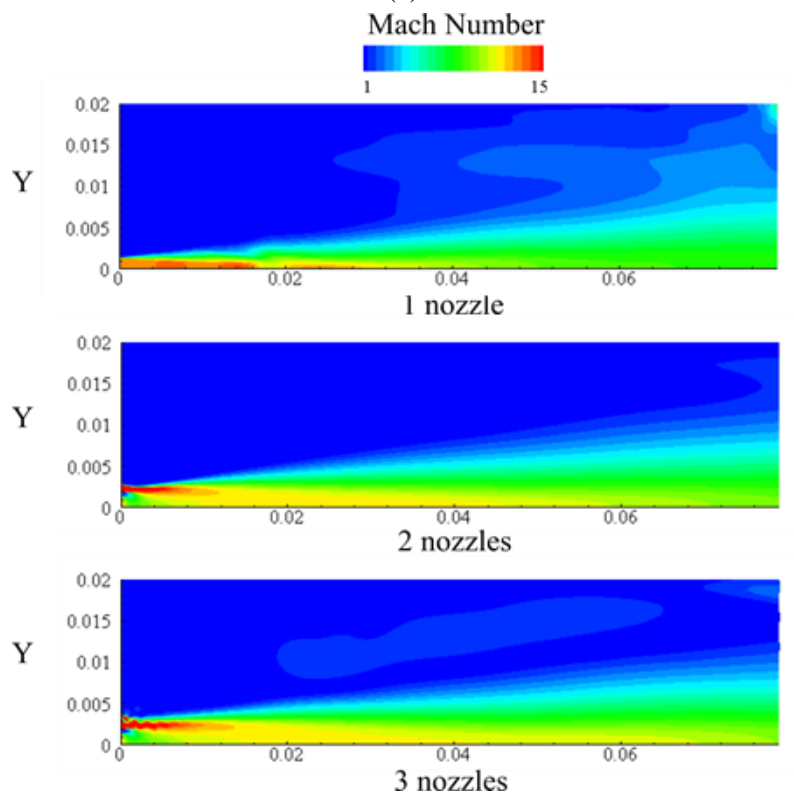

(b)

Fig. 4 Distributions of velocity magnitude and Mach number at non-dimensional time of $t^{*}=767$, (a) velocity magnitude and (b) Mach number.

relatively lower Mach numbers are more significant to suppress a tendency of overexpansion of the inner jet of nozzle 2. From the result, a role of the jet of nozzle 3 with an appropriate condition must be to adjust an ambient pressure (or static pressure of the jet of nozzle 3) for the inner jet of nozzle 2 to an optimum pressure and to suppress overexpansion of the jet form nozzle 2 . 

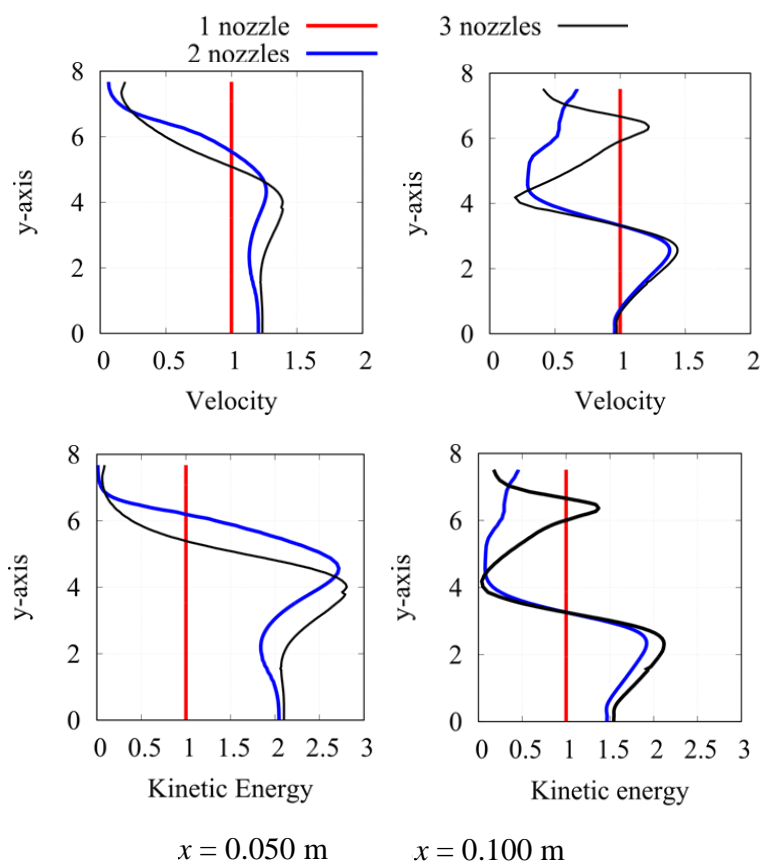

Fig. 5 Distributions of normalized velocity magnitude and kinetic energy normalized by the results of single nozzle case by Eqs. (1) and (2) for $x=0.050 \mathrm{~m}$ and $0.100 \mathrm{~m}$ downstream from the nozzles.

\subsection{The Effectiveness of a Plasmajet on Acceleration of a Coaxial Free Jet}

\subsubsection{Condition}

In this section, an investigation is conducted to assess the effectiveness of a plasmajet on acceleration of a coaxial free jet. A simulation model and outer boundary are as described in Chapter 2 .

An investigation is conducted on the influence of conditions of the plasmajet (nozzle 1) on acceleration of a coaxial supersonic free jet. The conditions of the nozzles are listed in Table 3, where heated plasmajet of nozzle 1 labeled as case1-1, and non-heated jet of nozzle 1 as case $1-2$.

Table 3 Numerical condition of case 1. nozzle 1

\begin{tabular}{lccccc}
\hline & & Mach number & $T_{0} \mathrm{~K}$ & $d_{\text {throat }} \mathrm{mm}$ & $\dot{m} \mathrm{slm}$ \\
\hline case1-1 & Plasma & 2.65 & 2000 & 1.5 & 24 \\
case1-2 & Air & & 300 & & 24 \\
\hline
\end{tabular}

nozzle 2,3

\begin{tabular}{lccccc}
\hline & & Mach number & $T_{0} \mathrm{~K}$ & $d_{\text {throat }} \mathrm{mm}$ & $\dot{m} \mathrm{slm}$ \\
\hline nozzle2 & Air & 2.0 & \multirow{2}{*}{300} & 0.65 & 308 \\
nozzle3 & Air & 1.7 & & 0.20 & 152 \\
\hline
\end{tabular}

\subsubsection{Results and Discussions}

Distributions of velocity magnitude and Mach number in the nearness of the nozzles at non-dimensional time of $t^{*}=767$ are shown in Figs. $6 \mathrm{a}$ and $6 \mathrm{~b}$, respectively.

From the figure, it is shown that higher velocities can be maintained up to downstream region in heated jet condition (case1-1) than non-heated jet condition (case1-2). On the other hand, as for Mach numbers, higher numbers can be maintained in non-heated jet condition (case1-2). Since a total temperature of the heated jet is higher (approximately 7 times) than that of non-heated jet, causing a much difference in speeds of sound at nozzle outlets in both cases, local static temperatures and speed of sounds of heated jet case (case1-1) are larger, then resulting in lower local Mach numbers than non-heated jet case (case1-2).

Distributions of normalized velocity and kinetic energy are plotted in Fig. 7, which are normalized by the results of non-heated jet case (case1-2) by Eqs. (1) and (2), at $x=0.100 \mathrm{~m}$ downstream from the nozzle. From the figure, it can be seen that higher velocities (about 3.5 times higher than non-heated jet case) are obtained from the center region to $y / d=3$. Since velocities of the non-heated jet case are smaller at downstream region, especially near the outer jet boundary, at $y / d=4.5$, effects of the heated jet on velocities are significantly enhanced by factor of 5 . As for normalized kinetic energies, higher kinetic energies (about 6 times higher) can be obtained from the center region to $y / d=3$. Because of the smaller kinetic energies of the non-heated jet at an outer jet boundary, at $y / d=4.5$, at downstream region, effects of the heated jet on increase of kinetic energies are significantly enhanced by factor of 14 .

From the results, it is shown that the heated jet at the center is more effective than a non-heated jet in acceleration of coaxial outer jets.

In addition, gradients of axial ( $x$-) component velocity in radial ( $y$-) direction, $\mathrm{d} u / \mathrm{d} y$, are considered. 


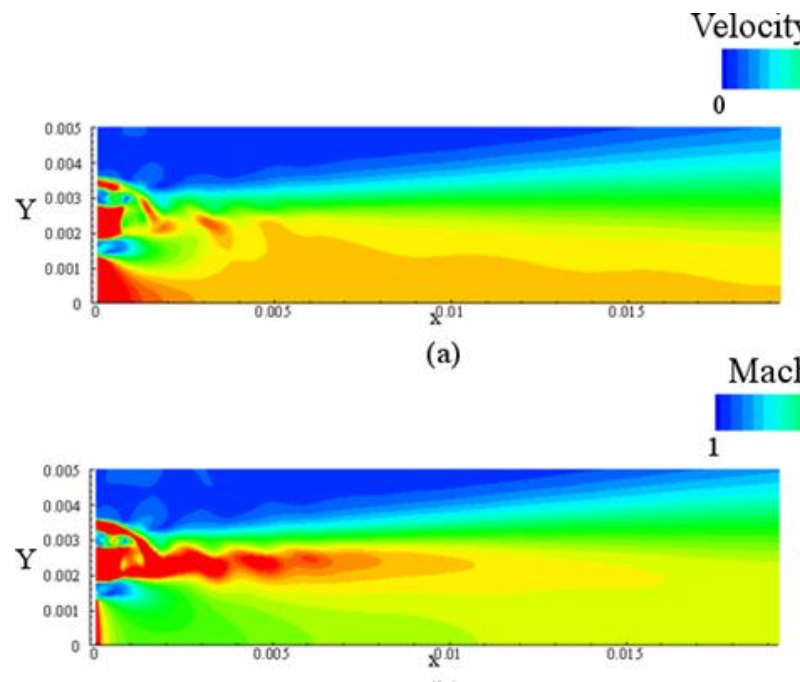

(b)

case1-1 Plasmajet flow elocity Magnitude

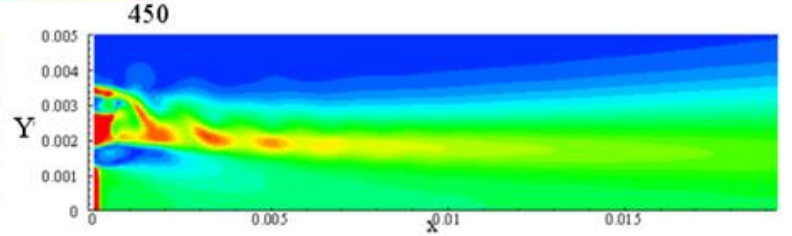

(a)
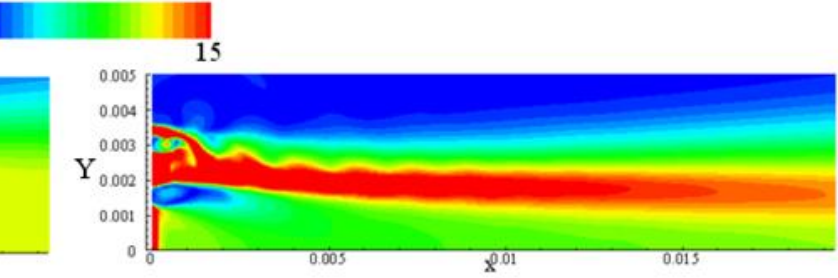

(b)

case1-2 Non-heated flow

Fig. 6 Distributions of velocity magnitude and Mach number at non-dimensional time of $t^{*}=767$, (a) velocity magnitude and (b) Mach number.
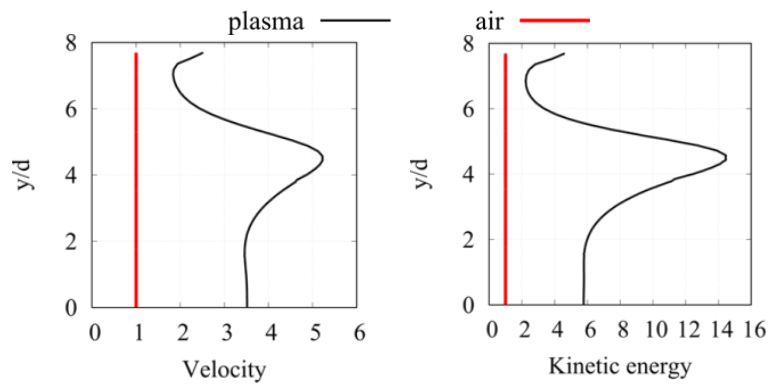

Fig. 7 Distributions of normalized velocity and kinetic energy normalized by the results of non-heated jet case (case1-2) by Eqs. (1) and (2) at $x=0.100 \mathrm{~m}$ downstream from the nozzle.

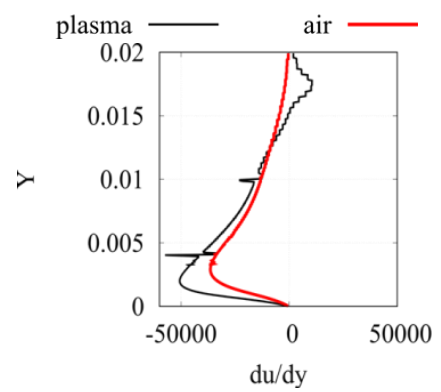

Fig. 8 Variations of the velocity gradients for heated and non-heated jet cases at $x=0.050 \mathrm{~m}$ downstream from the nozzle.

Fig. 8 shows variations of the velocity gradients for heated and non-heated jet cases at $x=0.050 \mathrm{~m}$ downstream from the nozzle. With the heated jet case, magnitudes of the velocity gradients become largest at $y=0$ to $0.002 \mathrm{~m}$, corresponding to a separation between a central heated jet (nozzle 1) and a coaxial outer jet (nozzle 2), or namely at a shear layer. Moreover, at $y=0.003$ to $0.004 \mathrm{~m}$, corresponding to another separation, or shear layer, between nozzle 2 and nozzle 3 , the values are large. On the other hand, in the non-heated jet case, tendencies are similar, however, their magnitudes are smaller than heated jet case.

Since local shear stresses acting on fluid elements are proportional to the local velocity gradients $(\mathrm{d} u / \mathrm{d} y)$, momentum and energy transfers in the shear layer from an inner heated jet to an outer jet in the heated jet case must be more efficient than those of non-heated case. Moreover, higher magnitudes of the velocity gradients must be more efficient in the momentum and energy transfers.

\subsection{The Influence of Total Temperatures of the} Plasmajet (Nozzle 1) on Acceleration of a Coaxial Supersonic Free Jet

\subsubsection{Condition}

In this section, the influence of total temperatures of the plasmajet (nozzle 1) on acceleration of a coaxial supersonic free jet is compared and discussed. A simulation domain and dimensions are similar to 
Table 4 Numerical condition of case 2.

nozzle 1

\begin{tabular}{lccccc}
\hline & & Mach number & $T_{0} \mathrm{~K}$ & $d_{\text {throat }} \mathrm{mm}$ & $\dot{m}$ slm \\
\hline case1-1 & Plasma & 2.65 & 2000 & 1.5 & 24 \\
\hline case2-1 & & & 1000 & & \\
case2-2 & Plasma & 2.65 & 3000 & 1.5 & 24 \\
case2-3 & & & 4000 & & \\
\hline
\end{tabular}

\begin{tabular}{lccccc}
\hline \multicolumn{6}{c}{ nozzle 2, 3 } \\
\hline \multicolumn{7}{c}{ Mach number } & $T_{0} \mathrm{~K}$ & $d_{\text {throat }} \mathrm{mm}$ & $\dot{m}$ slm \\
\hline nozzle2 & Air & 2.0 & \multirow{2}{*}{300} & 0.65 & 308 \\
nozzle3 & Air & 1.7 & & 0.20 & 152 \\
\hline
\end{tabular}

those employed and described in the previous section. The conditions of the central plasmajet are listed in Table 4, where different total temperatures of the heated plasmajet of nozzle 1 are labeled as case2-1 for $T_{0}=1,000 \mathrm{~K}$, case $2-2$ for $T_{0}=3,000 \mathrm{~K}$, and case2-3 for $T_{0}=4,000 \mathrm{~K}$.

\subsubsection{Results and Discussions}

Distributions of velocity magnitude and Mach number in the nearness of the nozzles at non-dimensional time of $t^{*}=767$ are shown in Figs. 9 and 10, respectively. From the figure, it is shown that higher velocities can be maintained further to downstream region in higher temperature cases of heated jet conditions. On the other hand, as for Mach numbers, higher numbers can be maintained to downstream region in lower temperature cases. In a case of the highest total temperature (case2-3), the largest values in magnitude of velocities can be maintained further to downstream region. As can be seen in these figures,

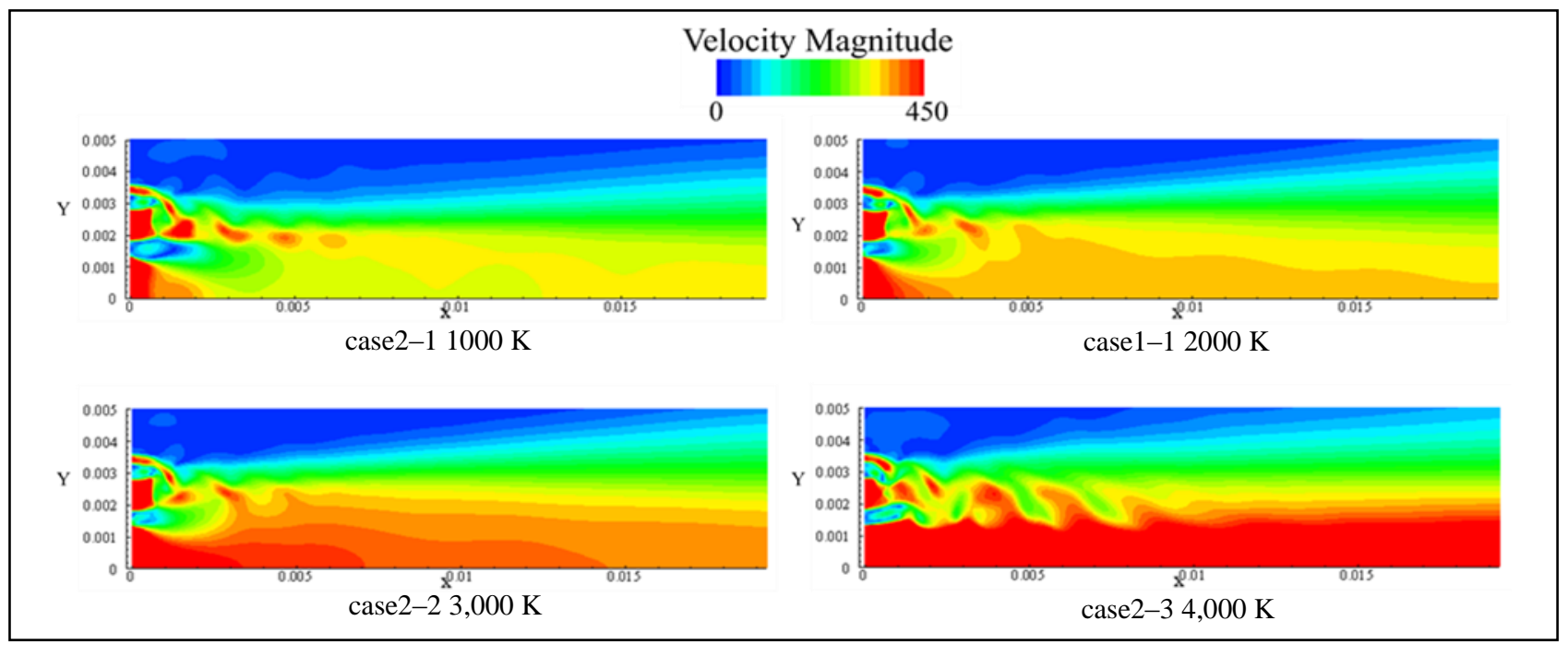

Fig. 9 Distributions of velocity magnitude at non-dimensional time of $t^{*}=767$ in case 2 .

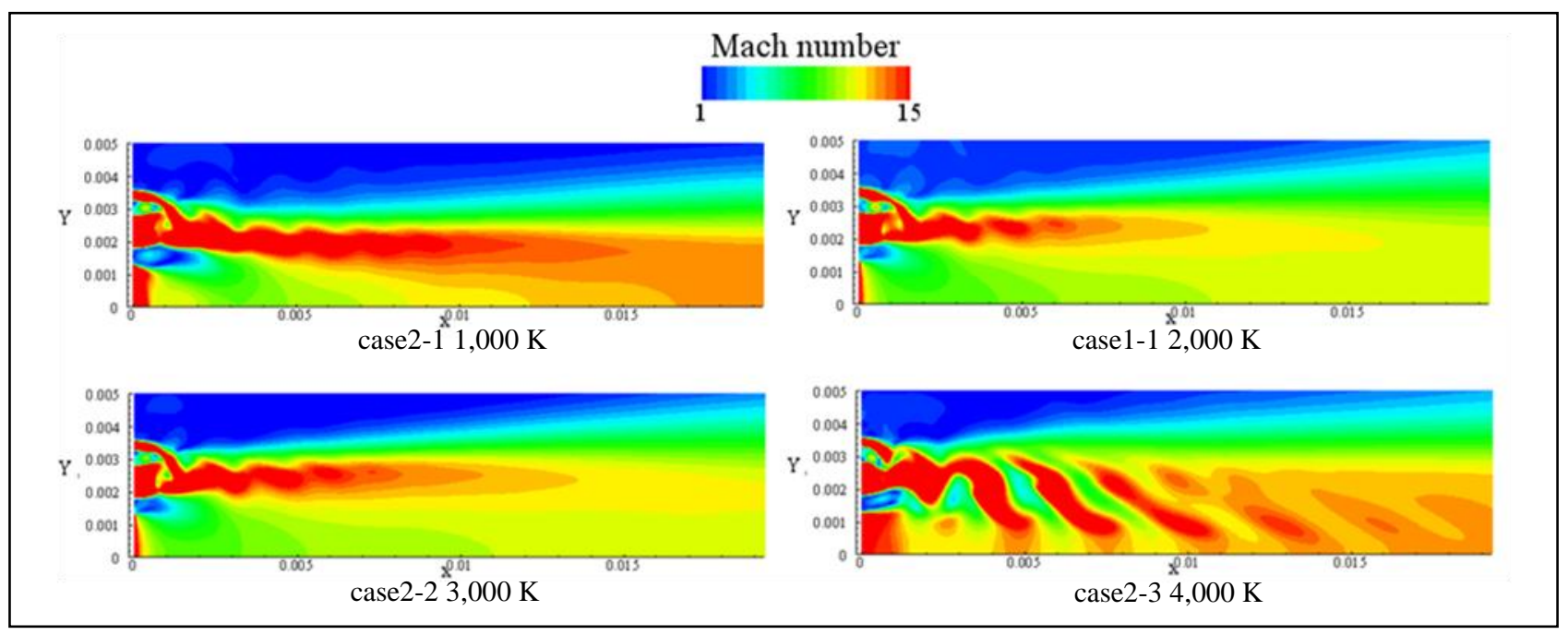

Fig. 10 Distributions Mach number at non-dimensional time of $t^{*}=767$ in case 2. 

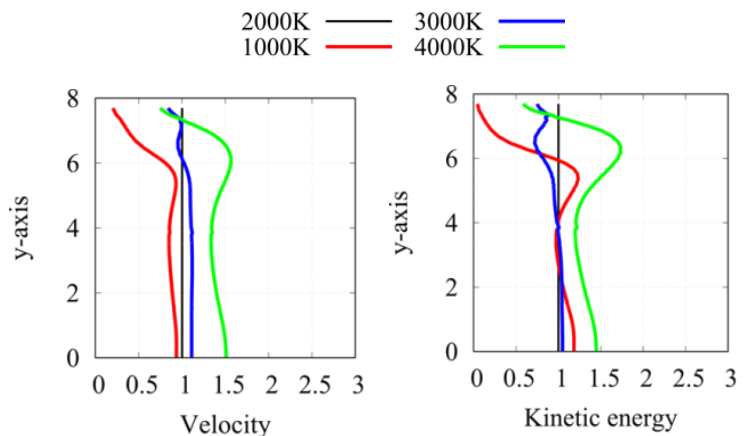

Fig. 11 Distributions of normalized velocity and kinetic energy normalized by the results of the temperature of $2,000 \mathrm{~K}$ case (case1-1) by Eqs. (1) and (2) at $x=0.100 \mathrm{~m}$ downstream from the nozzle.

since positions and strengths of normal or oblique shock waves in exhaust region of the plasmajet outlet are different with the increase of total temperature, influences of the shocks on the downstream flow are significantly different. In these figures, a significant drop in Mach number is occurring due to a normal shock at the nozzle exhaust region except case2-3 where oblique shocks and expansion fans are occurring alternatively and forming shock cell structures [12].

Distributions of normalized velocity and kinetic energy are plotted in Fig. 11, which are normalized by the results of heated jet case (case1-1, $T_{0}=2,000$ $\mathrm{K})$ at $x=0.100 \mathrm{~m}$ downstream from the nozzle. From the figure, it is shown that magnitudes of the normalized velocities become larger with the increase of total temperatures. This is simply due to the larger total enthalpies with higher temperature cases. Although differences of the normalized kinetic energies for total temperatures of $1,000 \mathrm{~K}, 2,000 \mathrm{~K}$ and $3,000 \mathrm{~K}$ are insignificant, significantly larger energies can be obtained with that of $4,000 \mathrm{~K}$ by factor of 1.5. Due to smaller kinetic energies of the lower temperature cases at outer jet boundary, at $y / d$ $=6$, at downstream region, effects of the total temperature on increase of kinetic energies are significantly enhanced by factor of 1.7.

From the results, it can be expected that further increase of the total temperature is more effective in acceleration of coaxial outer jets.

Comparing results of total temperatures of $2,000 \mathrm{~K}$ (case1-1) and 4,000 K (case2-3), although the total enthalpy is increased by factor of 2 , the gain of the kinetic energy is by factor of 1.5. Therefore, it appears that conversion efficiencies of the total enthalpies to kinetic energies are becoming suppressed with the increase of the total temperature due to the enhanced complexity of the flow.

Variations of velocity gradients $(\mathrm{d} u / \mathrm{d} y)$ are plotted in Fig. 12 for various total temperature cases at $x=$ $0.050 \mathrm{~m}$ downstream from the nozzle. From the figure, it is shown, in all cases, that magnitudes of the velocity gradients become largest at $y=0$ to $0.002 \mathrm{~m}$, corresponding to a boundary between a central heated jet (nozzle 1) and a coaxial outer jet (nozzle 2), or namely at a shear layer. Moreover, relatively large velocity gradients are given at $y=0.003$ to $0.004 \mathrm{~m}$, corresponding to another boundary, or shear layer, between nozzle 2 and nozzle 3 . In addition, the magnitudes of the velocity gradients become greater with the increase of total temperatures. Since local shear stresses acting on fluid elements depend on local velocity gradients $(\mathrm{d} u / \mathrm{d} y)$, it is shown that momentum and energy transfers in the shear layer from an inner heated jet to an outer jet become more effective with elevated temperatures. Larger magnitudes of the velocity gradients must be more efficient in the

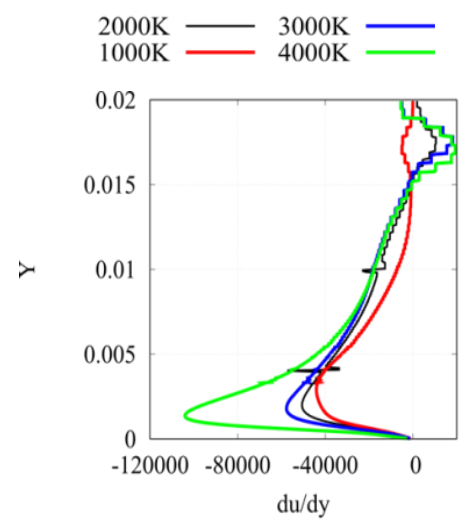

Fig. 12 Variations of the velocity gradients for heated and non-heated jet cases at $x=0.050 \mathrm{~m}$ downstream from the nozzle. 
momentum and energy transfers, or namely acceleration of the flow.

\subsection{The Effect of Mach Numbers of Plasmajets}

\subsubsection{Condition}

In this section, the influence of Mach numbers at outlet of the plasmajet (nozzle 1) on acceleration of a coaxial supersonic free jet is assessed. A simulation domain and dimensions are similar to those employed and described in the previous sections.

The conditions of the central plasmajet (nozzle 1) and coaxial jets (nozzle 2, 3) are listed in Table 5, where different Mach numbers of the heated plasmajet of nozzle 1 are labeled as case $3-1$ for $M=$ 1.8, case3-2 for $M=1.9$, case3-3 for $M=2.0$, and case1-1 for $M=2.65$.

\subsubsection{Results and Discussions}

Distributions of Mach number at non-dimensional time of $t^{*}=767$ are shown in Fig. 13. From the figure, it is shown that higher Mach numbers can be maintained to downstream region in smaller Mach number cases of heated jets outlets. This is probably because of the suppressed strengths of shocks, or namely reduced magnitudes of losses, with the decrease of Mach numbers of the plasmajets (nozzle 1). Distributions of normalized velocity and kinetic energy are shown in Fig. 14, which are normalized by the results of heated jet case (case1-1, $M=2.65$ ) at $x=0.100 \mathrm{~m}$ downstream from the nozzle. From the figure, it is shown that magnitudes of the normalized velocities and kinetic energies are relatively large at near centerline region. Moreover, comparing the results with those of $M=2.65$, smaller Mach number of the plasmajet, i.e. $M=1.8$ to 2.0 , or $M=1.9$, is more effective to obtain larger velocities and energies. However, their differences are insignificant.

Variations of velocity gradients $(\mathrm{d} u / \mathrm{d} y)$ are shown in Fig. 15 for various Mach number cases of the plasmajet at $x=0.050 \mathrm{~m}$ downstream from the nozzle.

From the figure, it is shown, in all cases, that magnitudes of the velocity gradients become largest at $y=0$ to $0.002 \mathrm{~m}$, corresponding to a boundary between a central heated jet (nozzle 1) and a coaxial outer jet (nozzle 2), or namely at a shear layer. Moreover, relatively large velocity gradients are obtained at $y=0.003$ to $0.004 \mathrm{~m}$, corresponding to another boundary, or shear layer, between nozzle 2

Table 5 Numerical condition of case 3. nozzle1

\begin{tabular}{lccccc}
\hline & & Mach number & $T_{0} \mathrm{~K}$ & $d_{\text {throat }} \mathrm{mm}$ & $\dot{m}$ slm \\
\hline case1-1 & Plasma & 2.65 & 2000 & 1.5 & 24 \\
\hline case3-1 & & 1.8 & & 2.15 & 50 \\
case3-2 & Plasma & 1.9 & 2000 & 2.10 & 47 \\
case3-3 & & 2.0 & & 2.00 & 43 \\
\hline
\end{tabular}

nozzle 2,3

\begin{tabular}{lccccc}
\hline & & Mach number & $T_{0} \mathrm{~K}$ & $d_{\text {throat }} \mathrm{mm}$ & $\dot{m}$ slm \\
\hline nozzle2 & Air & 2.0 & \multirow{2}{*}{300} & 0.65 & 308 \\
nozzle3 & Air & 1.7 & & 0.20 & 152 \\
\hline
\end{tabular}

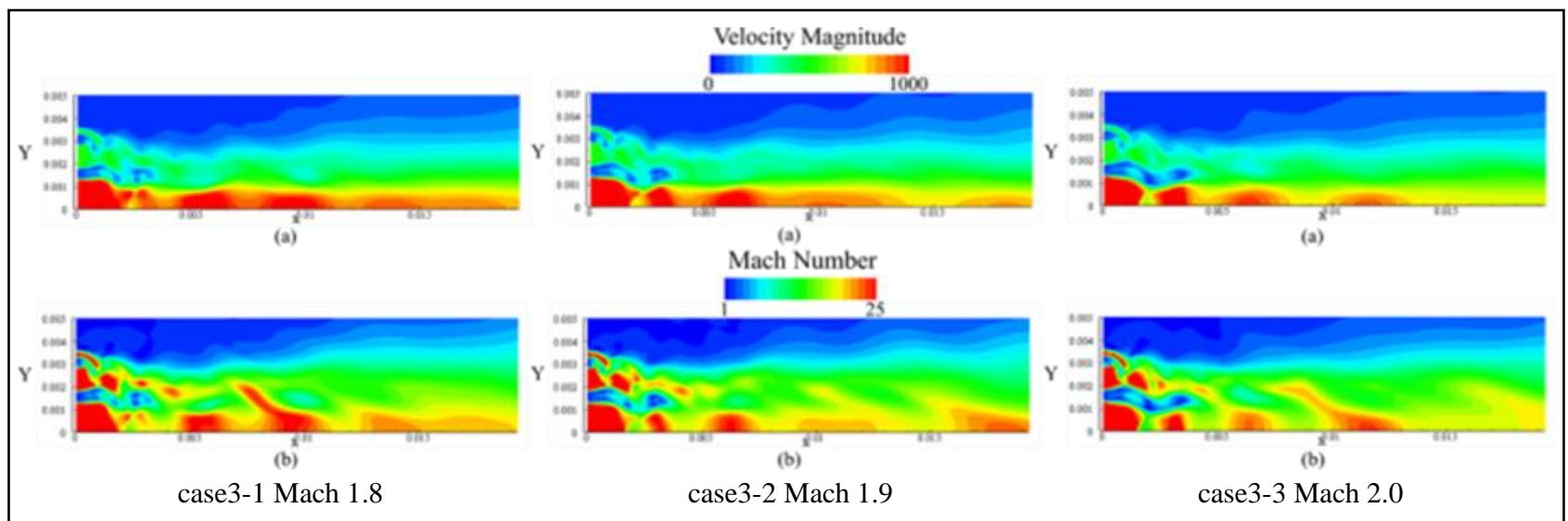

Fig. 13 Distributions of velocity magnitude and Mach number at non-dimensional time of $t^{*}=767$, (a) velocity magnitude and (b) Mach number in case 3. 

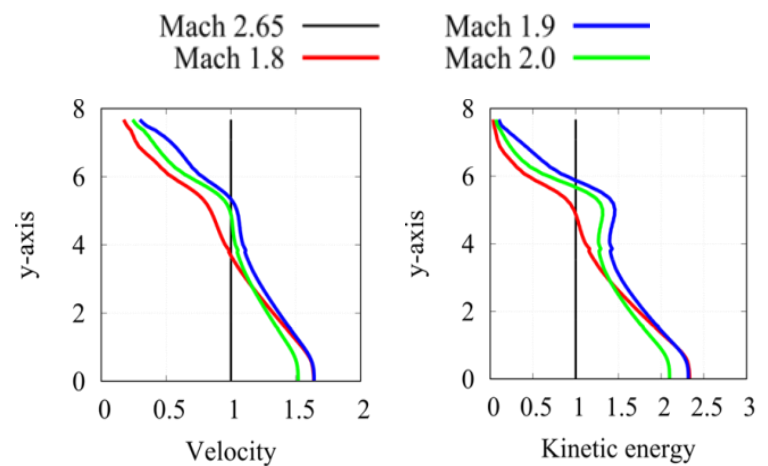

Fig. 14 Distributions of normalized velocity and kinetic energy normalized by the results of the temperature of $M=$ 2.65 case (case1-1) by Eqs. (1) and (2) at $x=0.100 \mathrm{~m}$ downstream from the nozzle.

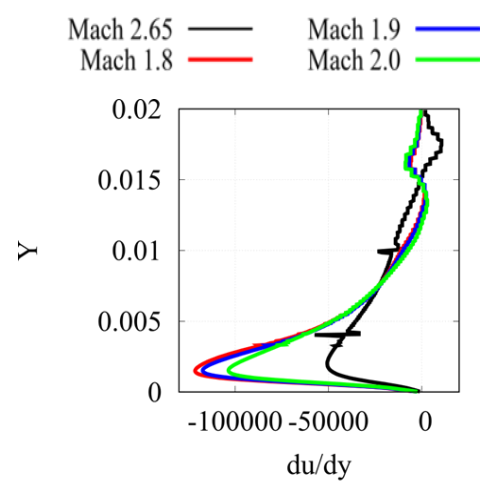

Fig. 15 Variations of the velocity gradients for heated and non-heated jet cases at $x=0.050 \mathrm{~m}$ downstream from the nozzle.

and nozzle 3. In addition, the magnitudes of the velocity gradients become larger with the reduction of Mach numbers of the central heated jet (nozzle 1). Larger magnitudes of the velocity gradients must be more efficient in the momentum and energy transfers, or namely acceleration of the flow. Therefore, it appears that smaller Mach numbers of the plasmajet are more effective in acceleration of the coaxial jets.

\section{Conclusions}

In this study, numerical simulation on acceleration characteristics of a supersonic free jet through utilization of a plasmajet was conducted, where the supersonic free jet was accelerated through an interaction in a shear layer between the free jet and plasmajet. All in the results obtained:

A role of the jet of nozzle 3 with an appropriate condition must be to adjust an ambient pressure (or static pressure of the jet of nozzle 3) for the inner jet of nozzle 2 to an optimum pressure and to suppress overexpansion of the jet from nozzle 2 .

Momentum and energy transfers in the shear layer in the heated jet case must be more efficient than those of non-heated case.

Further increase of the total temperature of the plasmajet was more effective in acceleration of coaxial outer jets.

Smaller Mach numbers of the plasmajet were more effective in acceleration of the coaxial jets.

\section{References}

[1] Goethert, B. H., and Matz, R. 1964. "Experimental Investigation of Base Flow Characteristics of Four-Nozzle Cluster-Rocket Models." ARO, Inc., No. AF40 (600)-1000.

[2] Murthy, S. N. B. 1975. Turbulent Mixing in Nonreactive and Reactive Flows. Plenum Press.

[3] NASA Langley Research Center. 1972. "Free Turbulent Shear Flows." NASA-SP-321.

[4] Roshko, A. 1976. "Structure of Turbulent Shear Flows: A New Look." AIAA Journal 14 (10).

[5] Sutton, G. P., and Biblarz, O. 2016. Rocket Propulsion Elements. John, Wiley Interscience Publication.

[6] Jahn, R. G. 2006. Physics of Electric Propulsion. Dover Publications.

[7] Salonitisa, K., and Vatousianos, S. 2012. "Experimental Investigation of the Plasma Arc Cutting Process." CIRP, 287-92.

[8] Bhowmick, S., Basu, J., Majumdar, G., and Bandyopadhyay, A. 2018. "Experimental Study of Plasma Arc Cutting of AISI 304 Stainless Steel." ICMPC 5 (2): 4541-50.

[9] Scortecci, F., d'Agostino, L., d'Auria, F., and Andrenucci, M. 1993. "Arcjet Propulsion System Study for NSSK." University of Pisa. IEPC, 93-013.

[10] Sleziona, P. C., Auweter-Kurtz, M., Glocker, B., Gogel, T., Golz, T., Messerschmid, E., and Schrade, H. O. 1991. "Non-equilibrium Flow in an Arc Heated Wind Tunnel." In Hypersonic Flows for Reentry Problems, University of Stuttgart, pp. 1116-30.

[11] Brescianini, C. P. 1992. "Modified k-ipsiron Model for Compressible Shear Flows.” AIAA Journal 30 (8).

[12] Mehta, R. C., and Prasad, J. K. 1996. "Estimation of Shock-Cell Structure of Axisymmetric Supersonic Free Jets." Indian Journal of Engineering and Materials Sciences 3: 141-7. 